\title{
A License Plate Extraction Algorithm Based on Edge Statistics and Region Growing
}

\author{
Manuel Vargas, Sergio L. Toral, Federico Barrero, and Francisco Cortés \\ E. S. Ingenieros, University of Seville \\ Camino de los Descubrimientos s/n, 41092 Seville, Spain \\ \{vargas, toral, fbarrero, fcortes1\} @esi.us.es
}

\begin{abstract}
This paper presents a license plate extraction method for gray-scale images, based on a combination of edge statistics and a two-step seeded region growing algorithm. The proposed region growing algorithm uses a dual criterion based on edge density and gray-scale intensity affinity. The proposed method aims at achieving license plate segmentation that fits to the real plate boundaries better than existing methods. The robustness of the method has been tested with experimental results, including examples of low quality or poorpreserved plates and commercial or freight transport vehicles.
\end{abstract}

Keywords: Automatic License Plate Recognition (ALPR), plate localization, edge statistics, seeded region growing.

\section{Introduction}

Intelligent Transportation Systems (ITS) can be considered a global phenomenon, attracting worldwide interest from transportation professionals, the automotive industry, and political decision makers [1]. As a consequence, ITS involves a large number of research areas spread over many different technological sectors. One of the most important research issues is Automatic License Plate Recognition (ALPR), with important applications in areas like traffic surveillance, traffic control, vehicle tracking, localization, car park automation, electronic toll collection systems, and several other applications [2].

A typical ALPR system consists of three major phases: license plate detection, geometric correction, character segmentation, size or aspect ratio normalization, character recognition and application of grammatical rules [3], [4], [5]. Among them, a very critical step is the license plate location and segmentation, which directly affects the overall system performance.

A broad range of methods for license plate segmentation have been reported in the literature. A recent survey on this topic can be found in [6]. A first group of methods is based upon the combination of edge statistics and mathematical morphology [7], [8]. Other set of methods is based on color or gray-scale analysis [6]; they are based on scanning the image, looking for repeating contrast changes and counting edges [9]. In general, their performance and computational cost are worse than those provided by the first group of methods. Finally, classifiers based on statistical methods [10], 
artificial neural networks [11], genetic programming [12] and genetic algorithms [13] have also been proposed in the literature.

The approach followed in this paper can be included in the first group of methods, that is, making use of edge statistics for plate location, combined with a two-step region growing algorithm, based on a dual (edge density / intensity affinity) criterion, with the aim of achieving a more accurate plate segmentation. The first region growing step uses just a gradient density criterion, leading to a typically elliptical region located at the core of the license plate. A simple statistical analysis is performed over this inner region in order to model the distribution of the plate background gray-level intensities. The objective of the second stage is growing from the inner region to include the whole license plate, adding a luminance criterion that tries to evaluate the affinity of gray-level intensities of the neighboring pixels with respect to the modeled plate background. The proposed method provides an accurate extraction of the plate region, reducing the task of false candidate rejection in subsequent stages.

\section{License Plate Extraction Algorithm}

The license plate of a vehicle contains rich edge information. Edge-based methods are based on this fact and the assumption that the areas containing a license plate will correspond to regions with higher edge density. Nevertheless, many false candidates can be obtained, and therefore, it is necessary to apply some morphological stages to choose the right ones. This decision making process will be greatly improved if the regions extracted are the most similar possible to the real plate dimensions and shape. In this paper, a processing stage based on a two-step region growing routine is proposed to accurate extract the plate region. The complete set of steps of the proposed method will be next listed and explained.

\subsection{Vertical-Edge Image}

Vertical edges are most prominent in the license plate region due to the contrasting vertical strokes of plate characters. On the opposite, the rear or front vehicle areas around the plate predominantly includes horizontal edges [14]. That is the reason why a vertical edge image is calculated as the first stage of the proposed method. Fig. 1 demonstrates the effect of applying a vertical edge 3x3 Sobel operator over the input image.

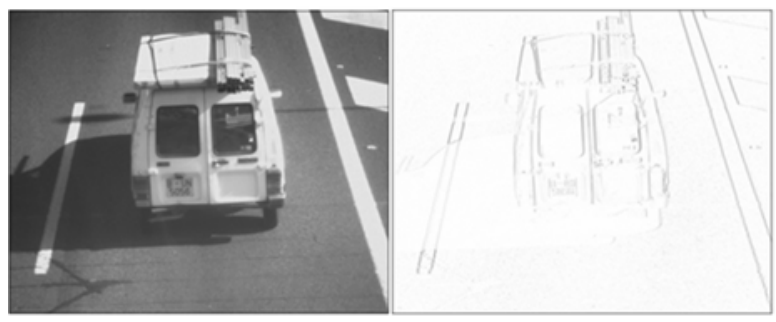

Fig. 1. Vertical-edge image 


\subsection{Binarization of Vertical-Edge Image}

The vertical-edge image has to be binarized to highlight those edges to be considered relevant. According to [15], the threshold is obtained from the histogram of the vertical-edge image, as the value that guarantees that a $\alpha \%$ of the image values are above the chosen threshold. This thresholding mechanism has the advantage of being selfadaptive to different illumination and contrast conditions in the scene. Values around 5 or 6 for this parameter, $\alpha$, have been empirically obtained, covering a wide range of illumination and contrast conditions. Fig. 2 shows the histogram and the resulting binarization, $B(x, y)=\{1,0\}$, for the previous vertical-edge image.
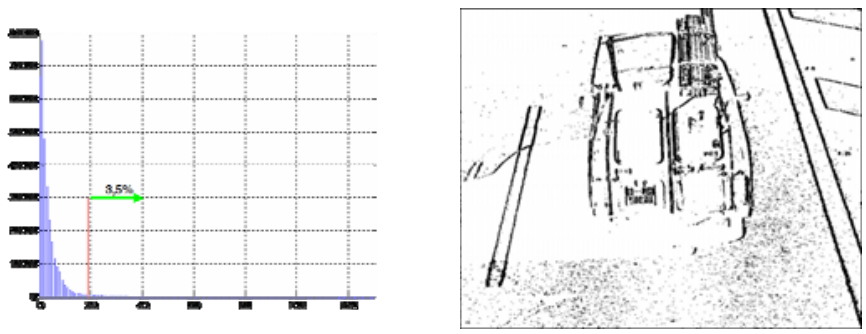

Fig. 2. Histogram and vertical-edge binarized image

\subsection{Candidate Extraction Using Vertical-Edge Density}

The region where the plate is located exhibits a high concentration of vertical edges. Consequently, a vertical-edge density image is next computed, in order to emphasize the location of candidate regions [16], [17].

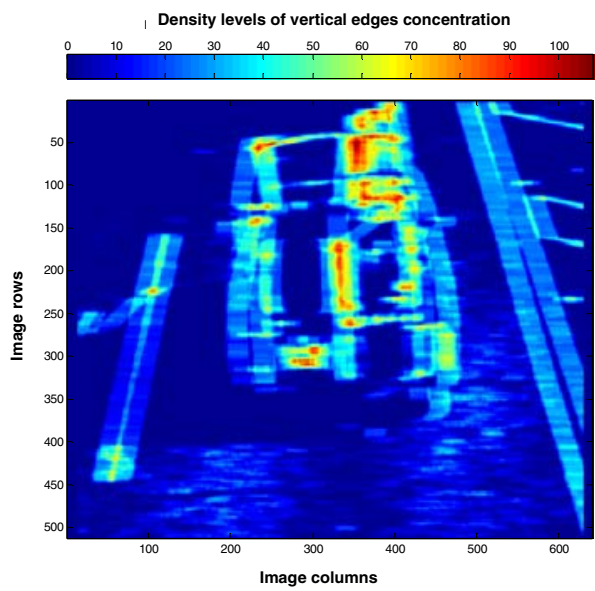

Fig. 3. Vertical-edge density image 
If we define a density mask of dimension $w x h$, a vertical-edge density image, $D(x, y)$, can be computed. The value of each pixel belonging to this image is calculated as the sum of the number of 1 -valued pixels in image $B(x, y)$ inside the window:

$$
D(x, y)=\sum_{i=x-w / 2}^{x+w / 2} \sum_{j=y-h / 2}^{y+h / 2} \frac{B(i, j)}{w \times h} \in[0,1]
$$

Fig. 3 illustrates the vertical-edge density image as a thermographic map, where "hot" areas denote high-density areas. Obviously, the obtained result critically depends on a proper selection of the density mask dimension. It should be chosen according to the size, inter-distance and aspect ratio of license plate characters. Given the maximum and minimum expected plate character heights, $h_{M}$ and $h_{m}$, respectively, and the character aspect ratio, $r$, the dimension of the density mask can be computed as:

$$
h=\frac{h_{M}+h_{m}}{2} \cdot 0.85 ; \quad w=h \cdot r \cdot 0.7
$$

This tuning rule has shown to work properly with very disparate values for expected character size, in particular: $h_{M}=33$ (pixels) and $h_{m}=10$ (pixels), while using a character aspect ratio: $r=0.58$, corresponding to the old and new fashioned Spanish car plates.

The density image is used to locate those regions which are likely to include a license plate. First, candidate rows are searched by performing a unidimensional analysis of the density map. In particular, the variance of the density values along each row are obtained, leading to the vector $v(y)$ depicted in Fig. 4. Notice the two peak values around row 300 corresponding to the square (two lines) vehicle license plate.

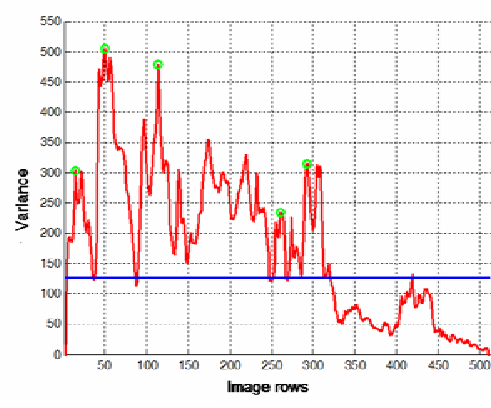

Fig. 4. Variance vector per rows of the vertical-edge density image

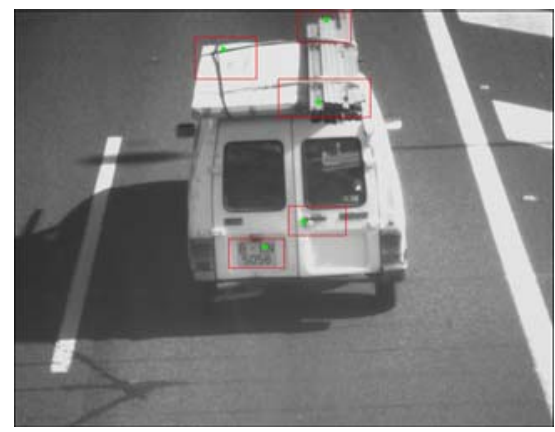

Fig. 5. Location of license plate candidates

Given the texture of license plates, the vertical-edge density, along a row belonging to a plate, typically exhibits higher variance values. Consequently, the relative maxima of the variance vector can be considered candidates for possible license plates. However, instead of considering all the relative maxima, a more selective procedure is followed. First, a set of intervals are defined (see Fig. 4) in the "image row" axis, 
then the absolute maximum inside each interval is taken as potential plate row (to be denoted as $r_{i}^{\text {cand }}$, for the $i$-th maximum). Each interval includes the set of adjacent rows whose variance is over a threshold, $\beta=k_{\beta} \max (v(y))$ (blue line in Fig. 4), given by a percentage of the global maximum variance.

The intervals around each $r_{i}^{\text {cand }}$ are then narrowed, in order to better fit to the plate vertical limits: $\left\lfloor r_{i}^{u p p}, r_{i}^{b o t}\right] . r_{i}^{u p p}$ is the first row towards the left of $r_{i}^{\text {cand }}$ that does not fulfill: $v\left(r_{i}^{u p p}\right)>k_{\delta} \cdot v\left(r_{i}^{\text {cand }}\right)$. Alternatively, $r_{i}^{\text {bot }}$ is the first row towards the right of $r_{i}^{\text {cand }}$ that does not fulfill the same condition.

For each candidate region, it is checked that its height is inside the range specified by the expected average size of the license plate characters.

A similar analysis is performed per columns, but only in those image stripes selected as possible candidates. Again, the relative maximum values of the variance $c_{i}^{c a n d}$ for each of the possible candidates are searched, and the left and right limit columns are obtained: $\left\lfloor c_{i}^{\text {left }}, c_{i}^{\text {right }}\right\rfloor$. Fig. 5 illustrates the resulting candidate set after the described per-row and per-column edge-density variance analysis. Typical values for parameters $k_{\beta}=0.15$ and $k_{\delta}=0.3$ (in the case of the analysis per columns: $k_{\beta}=0.2$ and $k_{\delta}=0.25$ ).

\subsection{Two-Step Region Growing}

For each candidate region obtained in the previous step, a seeded region growing algorithm is performed, using as the initial seed the maximum density point inside the candidate bounding box. This process consists of incorporating neighbour points fulfilling a similitude criterion. A two-step procedure with a combined similitude criterion is proposed to improve the segmentation of plate candidates.

The first step employs a criterion based on an upper density threshold for region growing. This threshold is defined as a certain percentage $\alpha_{1}$ of the maximum value corresponding to the initial seed. The result is a typically elliptic region centered on the license plate, but not reaching its limits.

As this region is expected to be located at the core of the plate, we will perform a simple luminance analysis, in order to classify those pixels owning to the background of the license plate from those belonging to the character strokes. On top of that, this analysis will allow distinguishing between white (light) background plates with black (dark) characters and the opposite.

The histogram of the previous region is obtained, $h(k)$. Then, on the one hand, the mean value of pixels' intensities $\mu$ is computed. On the other, an energy threshold, $\gamma$, is also computed, such that $75 \%$ of the histogram energy is under this threshold:

$$
\gamma=\underset{0 \leq i \leq 255}{\arg \min }\left[\sum_{k=0}^{i}\left(\frac{h(k)}{N}\right)^{2} \geq 0.75 \cdot E\right]
$$


where $N$ is the number of pixels of the analyzed region and $E$ is the total histogram energy: $E=\sum_{k=0}^{255}\left(\frac{h(k)}{N}\right)^{2}$

Fig. 6 illustrates an example of the referred histogram. Two vertical lines are marking the two thresholds considered, the intensity mean value $\mu$ and the energy threshold $\gamma$. If $\gamma>\mu$, the region labeled as "B" in Fig. 6 corresponds to the plate background which is a white background with dark characters. Otherwise, it means this region is the dark background with white characters. In any case, the intensity distribution of the plate background can be modeled assuming a Gaussian probability density function, $N\left(\mu^{B}, \sigma^{B}\right)$, where $\mu^{B}$ is the mean background intensity value, and $\sigma^{B}$ is the corresponding typical deviation.

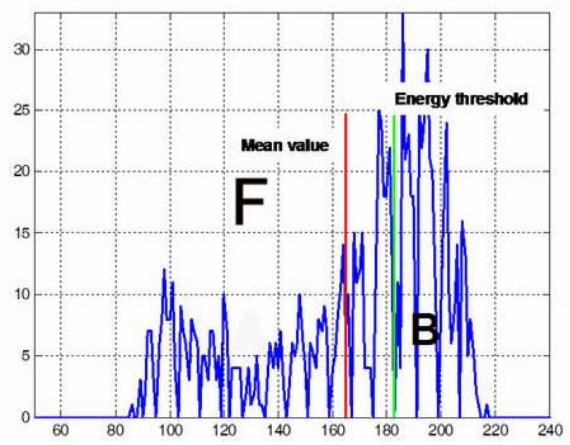

Fig. 6. Histogram of the resulting image after the first region growing process

The second stage of the region growing process consists of aggregating those pixels satisfying a combined criterion:

- Having a density level above a certain percentage $\alpha_{2}$ of the maximum value corresponding to the initial seed, which constitutes a lower density threshold $\left(\alpha_{2} \leq \alpha_{1}\right)$.

- Having an intensity level in an interval around the mean intensity value of pixels owning to the plate background. If $I^{B}(x, y)$ represents a plate background pixel, this can be described by:

$$
I^{B}(x, y) \in\left\lfloor\mu^{B}-k \cdot \sigma^{B}, \mu^{B}+k \cdot \sigma^{B}\right\rfloor
$$

Figures 7 and 8 illustrate two examples of the proposed two-step region growing algorithm ( $\alpha_{1}=49 \%, \alpha_{2}=31 \%$, have been used in these and next experiments). It can be verified that the bounding box of the obtained region accurately fits to the plate limits, even in cases of bright vehicles bearing plates with a bright background. 

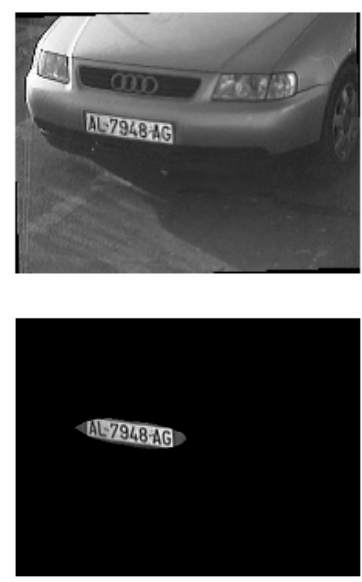
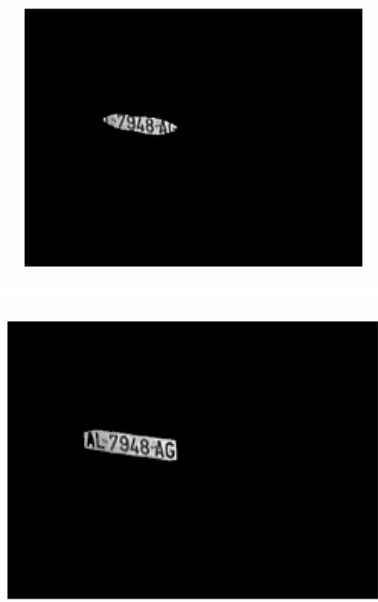

Fig. 7. From left to right and top to bottom: input image. First step (upper density threshold) region growing. Lower density threshold region growing. Two-step, combined-criterion (proposed) region growing.
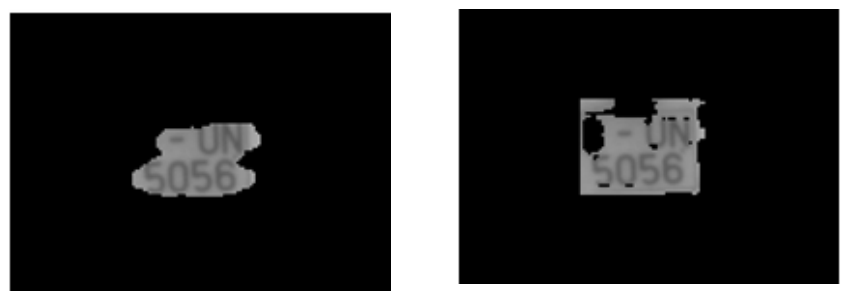

Fig. 8. From left to right: First step (upper density threshold) region growing. Two-step, combined-criterion (proposed), region growing.

\subsection{False Candidate Rejection}

During the region growing steps, several parameters can be simultaneously computed, which provide useful information for false candidate rejection: maximum and minimum inertia axes, rectangularity, aspect ratio, etc. Although some false candidates can be identified using this information, a more precise analysis is usually required, in order to reduce the false positive ratio. The extended analysis includes the binarization of the region and the extraction and analysis of connected components.

The binarization can be performed using a global or an adaptive threshold. In general, the adaptive solution is more computationally expensive, but provides better results [18]. Niblack [19] proposed computing the adaptive threshold for each pixel, $T(x, y)$, using the median and standard deviation of the neighbourhood intensity values. A variation of this method, introduced in [20] will be used, considering the difference between the maximum and minimum neighbouring intensities, instead of the standard deviation:

$$
T(x, y)=\mu(x, y)+k_{T} \cdot(M(x, y)-m(x, y))
$$



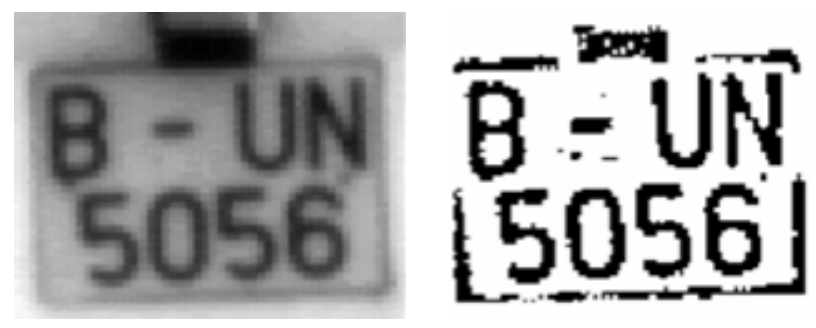

Fig. 9. Binarization of candidate regions using adaptative thresholding

Fig. 9 shows the binarization of one candidate region using the described adaptive thresholding. A connected component labeling is then applied to distinguish the license plate characters from other objects [21] (size, aspect ratio). Then, only the reliable labeled objects are used in order to test some conditions over the license plate candidate: collinearity of a minimum number of characters, minimum and maximum number of allowed characters in a legal plate, etc.

\section{Experimental Results}

Experimental results have been performed on an Intel Pentium Core2 Duo at 1,7 GHz, 1 GB RAM. Two image sets have been used. The first one corresponds to 243 good quality/contrast grayscale images. The second set of images is constituted by 100 low quality/contrast grayscale images. The main part corresponds to commercial or freight transport vehicles. In the latter case, many license plates are badlypreserved or partially covered by other elements. Besides, the presence of printed text on the vehicle makes more difficult the license plate location.

The following parameters have been measured (see [22]), in order to evaluate the performance of the proposed method:

- $\quad N R C$ : Number of raw candidates provided by the algorithm.

- $\quad N F C$ : Number of candidates validated as license plates. This parameter can be decomposed in true positives (TNFC) and false positives (FNFC).

- $\operatorname{MR}(\%)$ : Miss rate.

- $F D R(\%)$ : False detection rate.
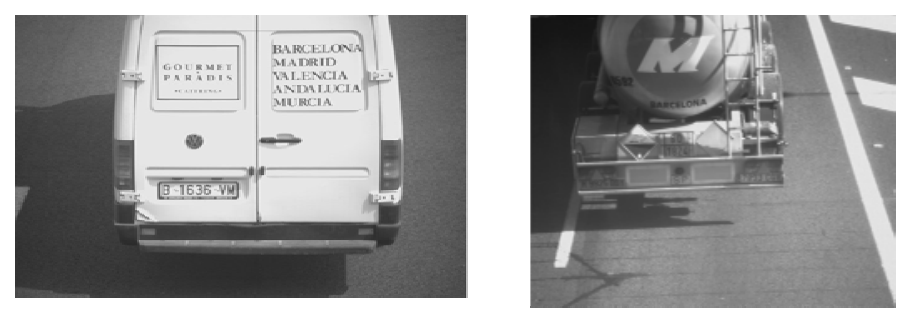

Fig. 10. Left: Sample image from test set 1. Right: Sample image from test set 2. 
Table 1. Metrics associated to two test sets

\begin{tabular}{|l|l|l|}
\hline & Set 1 & Set 2 \\
\hline NRC & 967 & 867 \\
\hline NFC & 241 & 108 \\
\hline TNFC & 236 & 92 \\
\hline FNFC & 5 & 16 \\
\hline MR $(\%)$ & 2,88 & 8 \\
\hline FDR $(\%)$ & 2,1 & 16 \\
\hline
\end{tabular}

Table 1 details the performance of the proposed method using the two set of images considered. The second set of images exhibits a miss and false detection rate much higher than the first one, as expected, due to the low quality of images and to the numerous commercial vehicles which usually includes text and publicity labels that are possible license plate candidates.

\section{Conclusions}

This paper reports a method for license plate segmentation. The main contribution of the paper is the proposed two-step region growing procedure, combining density and intensity image information, which allow achieving a better fit to the true license plate region. As reported in the experiments, the proposed method achieves a good extraction of license plates.

Acknowledgments. This work has been supported by the Spanish Ministry of Education and Science (Research Project with reference DPI2007-60128), the Consejería de Innovación, Ciencia y Empresa (Research Project P07-TIC-02621) and ACISA R\&D Department.

\section{References}

1. McQueen, B., McQueen, J.: Intelligent Transportation Systems Architectures. Artech House, Norwood (1999)

2. Walton, C.M.: The Heavy Vehicle Electronic License Plate Program and Crescent Demonstration Project. IEEE Transactions on Vehicular Technology 40(1), 147-151 (1991)

3. Caner, H., Gecim, H.S., Alkar, A.Z.: Efficient Embedded Neural-Network-Based License Plate Recognition System. IEEE Transactions on Vehicular Technology 57(5), 2675-2683 (2008)

4. Lopez, J.M., Gonzalez, J., Galindo, C., Cabello, J.: A versatile low-cost car plate recognition system. In: 9th Inl Symp. on Signal Processing and Its Applications ISSPA, pp. 1-4 (2007)

5. Comelli, P., Ferragina, P., Notturno Granieri, M., Stabile, F.: Optical Recognition of Motor Vehicle License Plates. IEEE Transactions on Vehicular Technology 44(4), 790-799 (1995) 
6. Anagnostopoulos, C.-N.E., Anagnostopoulos, I.E., Psoroulas, I.D., Loumos, V., Kayafas, E.: License Plate Recognition From Still Images and Video Sequences: A Survey. IEEE Transactions on Intelligent Transportation Systems 9(3), 377-391 (2008)

7. Wang, S., Lee, H.: Detection and recognition of license plate characters with different appearences. In: Proc. Conf. Intell. Transp. Syst., vol. 2, pp. 979-984 (2003)

8. Ma, Z., Yang, J.: A license plate locating algorithm based on multiple Gauss filters and morphology mathematics. In: Proc. 24th IASTED Int. Multiconference, Signal Process., Pattern Recog. Appl., Insbruck, Austria, February 15-17, 2006, pp. 90-94 (2006)

9. Wang, T.-H., Ni, F.-C., Li, K.-T., Chen, Y.-P.: Robust license plate recognition based on dynamic projection warping. In: Proc. IEEE Int. Conf. Netw., Sens. Control, pp. 784-788 (2004)

10. Wu, Q., Zhang, H., Jia, W., He, X., Yang, J., Hintz, T.: Car plate detection using cascaded tree-style learner based on hybrid object features. In: Proc. IEEE Int. Conf. AVSS, Sydney, Australia, pp. 15-20 (2006)

11. Chen, Y.-N., Han, C.-C., Wang, C.-T., Jeng, B.-S., Fan, K.-C.: The application of a convolution neural network on face and license plate detection. In: Proc. 18th ICPR, Hong Kong, vol. 3, pp. 552-555 (2006)

12. Adorni, G., Cagnoni, S., Mordonini, M.: Efficient low-level vision program design using sub-machine-code genetic programming. In: Proc. Workshop sulla Percezione e Visione nelle Machine (2002)

13. Cui, Y., Huang, Q.: Extracting characters of license plates from video sequences. Machine, Vision and Applications 10(5/6), 308-320 (1998)

14. Zheng, D., Zhao, Y., Wang, J.: An efficient method of license plate location. Pattern Recognition Letters 26(15), 2431-2438 (2005)

15. Martín Rodríguez, F., Fernández Hermida, X.: New Advances in the Automatic Reading of V.L.P.'s (Vehicle License Plates). In: Proceedings de SPC 2000 (Signal Processing and Communications), Marbella (September 2000)

16. Hongliang, B., Changping, L.: A hybrid License Plate Extraction Method based on Edge Statistics and Morphology. In: Proc. of the 17th IEEE Conference on Pattern Recognition (ICPR 2004), Cambridge, U.K. (2004)

17. Shapiro, V., et al.: Adaptive License Plate Image Extraction. In: Proc. of the Int. Conference on Computer Systems and Technologies (2003)

18. Feng, M.-L., Tan, Y.-P.: Contrast adaptive binarization of low quality document images. IECICE Electronics Express 1(6), 501-506 (2004)

19. Niblack, W.: An Introduction to Digital Image Processing. Prentice Hall, Englewood Cliffs (1986)

20. Wolf, C., Jolion, J.-M.: Extraction and Recognition of Artificial Text in Multimedia Documents. Pattern Analysis \& Applications 6(4), 309-326 (2003)

21. Chang, S.L., Chen, L.S., Chung, Y., Chen, S.: Automatic License Plate Recognition. IEEE Transactions on Intelligent Transportation Systems 5(1), 42-53 (2004)

22. Kim, K., Jung, K., Hyung, J.: Color Texture-Based Object Detection: An Application to License Plate Localization, pp. 293-309. Springer, Heidelberg (2002) 